\title{
PROPRIEDADES DE RESISTÊNCIA E RIGIDEZ DA MADEIRA JUVENIL E ADULTA DE Pinus taeda L. ${ }^{1}$
}

\author{
Adriano Wagner Ballarin² e Hernando Alfonso Lara Palma ${ }^{3}$
}

\begin{abstract}
RESUMO - O objetivo do presente trabalho foi o estudo da variabilidade das propriedades de resistência e rigidez à flexão estática e à densidade aparente (12\%) entre a madeira juvenil e adulta de Pinus taeda L., de 37 anos de idade, procedente do Horto Florestal de Manduri, Estado de São Paulo. Na primeira parte do trabalho foram determinadas a região de madeira juvenil, a região de transição e a região de madeira adulta, por meio de estudos anatômicos (comprimento dos traqueídes axiais), segundo as recomendações das normas ABNT e IAWA. Os resultados mostraram que a região de madeira juvenil dessa espécie ocorre aproximadamente até o $18^{\circ}$ anel de crescimento. Na segunda parte do trabalho foram analisados a resistência (módulo de ruptura - MOR) à flexão, o módulo de elasticidade (MOE) nessa mesma solicitação e a densidade aparente (12\%) para as madeiras juvenil e adulta. Os resultados mostraram que o MOE e o MOR da madeira juvenil foram menores e mais variáveis que aqueles obtidos para madeira adulta. A densidade apresentou a mesma tendência observada nas propriedades avaliadas no ensaio de flexão estática.
\end{abstract}

Palavras-chave: $\quad$ Madeira juvenil, madeira adulta, módulo de ruptura, módulo de elasticidade e Pinus taeda L.

\section{RIGIDITY AND STRENGTH PROPERTIES OF Pinus taeda L. JUVENILE AND MATURE TIMBER}

\begin{abstract}
This paper aimed to study the variability on rigidity (MOE) and strength (MOR) properties in static bending and density (12\%) between juvenile and mature wood of 37-year-old Pinus taeda L. trees from Horto Florestal, Manduri, Sao Paulo, Brazil. In the first part of the experimental program, the juvenile, transition and mature wood zones were determined using anatomical analysis (tracheids length) according to the ABNT and IAWA codes. Results showed that juvenile wood zone occurs approximately up to the $18^{\text {th }}$ growth ring. In the second part of the experimental setup, bending tests were performed with both juvenile and mature wood samples gathered in two distinct groups to obtain their modulus of rupture - MOR, modulus of elasticity -MOE and density (12\%). Results showed that MOE and MOR of juvenile wood were always smaller and presented more variability, when compared to mature wood. Density at $12 \%$ showed the same tendency observed in the mechanical tests.
\end{abstract}

Key words: $\quad$ Juvenile wood, mature wood, modulus of rupture, modulus of elasticity and Pinus taeda L.

\section{INTRODUÇÃO}

No passado, a maior parte da madeira provinha de árvores adultas de florestas naturais. Portanto, pouca importância era dada à madeira central das árvores.
Atualmente, com o decréscimo constante do suprimento de árvores adultas com grandes diâmetros, provenientes de florestas naturais, tornou-se comum a produção de madeira em ciclos curtos, através da adoção de espécies de rápido crescimento.

1 Recebido para publicação em 25.4.2002.

Aceito para publicação em 12.5.2003.

2 Dep. de Engenharia Rural da Universidade Estadual Paulista - UNESP, Fazenda Lageado s/n, Caixa Postal 237, $18603-970$ Botucatu-SP, <awballarin@fca.unesp.br>. ${ }^{3}$ Dep. de Recursos Naturais da UNESP, <larapalma@fca.unesp.br>. 
Existem várias referências na literatura internacional que ressaltam que as propriedades químicas, físicas, anatômicas e mecânicas da madeira formada nos primeiros anos de vida das árvores são diferentes e muitas vezes inferiores às da madeira formada na fase adulta da árvore.

No Brasil, a utilização de Pinus (de reflorestamentos) na indústria madeireira tem sido crescente nos últimos anos. As estimativas indicam que do volume de madeira serrada produzida no País, estimada em aproximadamente 18 milhões de $\mathrm{m}^{3}$, mais de $35 \%$ é formado de madeira de Pinus. Atualmente, no Brasil, existe aproximadamente 1,8 milhão de hectares de plantações constituídas por espécies de Pinus, das quais 46\% são de Pinus taeda (Tomaselli, 1998). Portanto, trata-se de uma espécie importante para o fornecimento de matériaprima, especialmente nas Regiões Sul e Sudeste do País.

O objetivo do presente trabalho foi o estudo da variabilidade das propriedades de resistência e rigidez à flexão estática e da densidade aparente (12\%) entre a madeira juvenil e adulta de Pinus taeda L., de 37 anos de idade, procedente do Horto Florestal de Manduri, Estado de São Paulo.

\section{REVISÃO DE LITERATURA}

A madeira juvenil corresponde a uma região central na árvore de forma cilíndrica, com diâmetro mais ou menos uniforme, estendendo-se desde a base até o topo da árvore, podendo formar parte do alburno ou do cerne no tronco, se este último já estiver presente na árvore (Krahmer, 1986; Zobel \& Buijtenen, 1989; Cown, 1992; Evans et al., 2000).

Segundo Ramsay \& Briggs (1986), a madeira juvenil é o xilema secundário, formado durante a fase jovem do câmbio vascular da árvore (estádios iniciais da vida da árvore). Este período varia conforme a espécie e pode ser afetado pelas condições ambientais. A madeira caracteriza-se anatomicamente por um progressivo acréscimo nas dimensões das células e por correspondentes alterações na sua forma, estrutura e disposição em sucessivos anéis de crescimento.

De modo geral, a maioria dos trabalhos sobre madeira juvenil enfatiza que o crescimento rápido nas plantações origina a formação de madeira de qualidade inferior e que, atualmente, é grande a proporção de madeira juvenil comercializada nos mercados, trazendo como resultado problemas de qualidade nos produtos obtidos deste tipo de matéria-prima (Brown \& Mcwilliams, 1989).

De acordo com Zobel (1981) e Mcalister et al. (1997), nas florestas naturais de climas temperados a madeira juvenil normalmente fica restrita a uma pequena região do volume total da árvore, e nas plantações a quantidade de madeira juvenil é maior, especialmente naquelas árvores provenientes dos primeiros desbastes ou de plantações cortadas mais cedo. Este fenômeno é muito mais acentuado nas plantações das regiões tropicais.

Zobel (1980) ressaltou que não há uma mudança absoluta da madeira juvenil para madeira adulta em um ano, mas sim em vários anos. Quase todas as propriedades físicas e químicas da madeira, dentro da zona juvenil, são muito variáveis e praticamente constantes na madeira adulta.

Muitos estudos têm sido conduzidos para determinar o ponto ou a idade de transição entre a madeira juvenil e a adulta em várias espécies, como: Loo et al. (1985) em Pinus sp., Bendtsen \& Senft (1986) em Pinus taeda e Populus deltoides, Yang et al. (1986) em Larix laricina, Clark \& Saucier (1989) em Pinus taeda e Pinus elliottii, Roos et al. (1990) em Populus tremuloides, Abdel-Gadir \& Krahmer (1993) em Pseudotsuga menziesii, Yang \& Hazenberg (1994) em Picea mariana e Tasissa \& Burkhart (1998) em Pinus taeda.

Segundo Zobel (1980) e Krahmer (1986), o comprimento dos traqueídes ou das fibras constitui a principal variável na definição do limite entre a madeira adulta e juvenil. Os traqueídes são mais curtos na região de madeira juvenil que na madeira adulta, e muito mais curtos perto da medula, aumentando rapidamente na zona de madeira juvenil em direção à casca. Mudanças no comprimento dos traqueídes na zona adulta da árvore são muito pequenas.

Segundo Zobel \& Buijtenen (1989), a mudança de madeira juvenil para madeira adulta não se processa da mesma forma para as diferentes características e propriedades da madeira. Por exemplo, a estabilização e a mudança se dão de maneira mais rápida para a densidade do que para o comprimento dos traqueídes.

A demarcação entre lenho juvenil e adulto não é clara, devido às mudanças graduais nas células. De fato, o número real de anéis do lenho juvenil depende de como ele é definido anatomicamente, por exemplo, o comprimento dos traqueídes pode atingir uma estabilidade antes da espessura da parede celular (Bendtsen \& Senft, 1986). 
Loo et al. (1985) reportaram que a idade de transição da madeira juvenil para adulta, mensurada através das variações de densidade ou do comprimento das fibras, é diferente de acordo com as espécies. Por exemplo, essa transição, avaliada pela variação de densidade, ocorre entre 5 e 6 anos em Pinus elliottii, Pinus caribaea e Pinus radiata, aproximadamente aos 10 anos em Pinus taeda e 20 anos em Pinus ponderosa.

Zobel (1971) e Kramher (1986) caracterizaram a madeira juvenil e adulta com base na evolução dos elementos anatômicos que as constituem; neste caso a madeira adulta refere-se ao lenho que apresenta traqueídes estabilizados em crescimento. Para o Pinus taeda com 30 anos de idade, essa estabilização foi encontrada a partir do 11 ao $13^{\circ}$ anel de crescimento.

Pesquisadores têm reportado a dificuldade na determinação precisa e consistente do limite entre a madeira juvenil e adulta, principalmente devido à transição gradual desta mudança e às variações existentes entre as espécies e a localização geográfica (Bendtsen \& Senft, 1986; Clark \& Saucier, 1989; Roos et al., 1990; AbdelGadir \& Krahmer, 1993; Evans et al., 2000).

A madeira juvenil, de modo geral, caracteriza-se por menor densidade, maior ângulo das microfibrilas na camada $S_{2}$, traqueídes mais curtos, contração transversal menor, maior contração longitudinal, maior proporção de lenho de reação, menor porcentagem de lenho tardio, paredes celulares mais finas, maior conteúdo de lignina e hemicelulose, menor conteúdo de celulose e menor resistência, em relação à madeira mais adulta (Bendtsen, 1978; Zobel, 1984; Senft et al., 1985; Rowell et al., 2000).

Peças estruturais que contenham uma determinada quantidade de lenho juvenil apresentam classes de resistência inferiores, sendo este o motivo pelo qual as diferenças entre as propriedades do lenho juvenil e adulto são importantes para a utilização da madeira (McAlister \& Clark, 1991; Geimer et al., 1997).

Segundo Larson et al. (2001), os módulos de elasticidade (MOE) e de ruptura (MOR) da madeira são altamente correlacionados com a densidade, portanto são influenciados pela qualidade da madeira juvenil. Os autores relataram que existem poucos estudos comparativos entre estas propriedades nos dois tipos de lenho. Os trabalhos encontrados na literatura apresentam valores baixos para a madeira juvenil, nestas duas propriedades.
As pesquisas encontradas neste sentido têm reportado que o módulo de elasticidade e a resistência à compressão paralela e normal, a flexão estática e a tração paralela são seriamente afetadas pela presença de madeira juvenil (Person \& Ross, 1984; Bentdsen \& Senf, 1986; Pearson, 1988; Kretschmann \& Bendtsen, 1992; Kretschmann, 1997; McAlister et al., 1997; Evans et al., 2000).

Outros estudos demonstram diferenças significativas entre a densidade da madeira juvenil e adulta em coníferas. Bendtsen (1978), em Pinus caribaea, encontrou densidades em torno de 0,36 e $0,68 \mathrm{~g} / \mathrm{cm}^{3}$ para os lenhos juvenil e adulto; Clark et al. (1989), em Pinus taeda, com 28 anos e em diferentes localidades geográficas, encontraram uma diferença que foi de 14 a $21 \%$ menor na madeira juvenil; McAlister \& Clark (1991), em Pinus taeda com 36 anos de idade e três localidades distintas, encontraram uma diferença de 22 a $28 \%$ menor entre as densidades da madeira juvenil; Yang \& Hazengerg (1994), em Picea mariana com 38 anos de idade, determinaram uma diferença $8 \%$ maior na densidade da madeira juvenil, em árvores com espaçamento inicial de 1,8 x 1,8 m em relação a espaçamentos maiores; e Kretschmann (1997), em Pinus taeda com 28 anos de idade, encontrou uma diferença $15 \%$ menor na madeira juvenil.

A produção de um tipo de madeira juvenil é o resultado do processo de crescimento fisiológico normal da árvore, portanto não há muitas alternativas para os silvicultores. A quantidade de madeira juvenil, que pode ser reduzida pela mudança no modelo de crescimento das árvores, é muito pequena. Quase todas as alternativas para conseguir maior crescimento nas árvores resultam em maiores quantidades de madeira juvenil. Por exemplo, quando as árvores de Pinus resinosa são fertilizadas com nitrogênio, a região de madeira juvenil aumenta no tronco (Zobel \& Buijtenen, 1989).

\section{MATERIAL E MÉTODOS}

A madeira utilizada neste trabalho foi obtida de árvores de Pinus taeda L., provenientes de plantios de 37 anos de idade, localizados no Horto Florestal de Manduri-SP (latitude $23^{\circ} 00^{\prime}$ sul, longitude $40^{\circ} 19^{\prime}$ oeste e altitude de $700 \mathrm{~m}$ ).

Foram selecionadas, aleatoriamente, seis árvores de um talhão de 1,6 ha, plantadas com um espaçamento inicial de $1,50 \times 1,50 \mathrm{~m}$, tendo sido realizados cinco

R. Árvore, Viçosa-MG, v.27, n.3, p.371-380, 2003 
desbastes até o corte final. A altura comercial e o DAP médio avaliados foram de $18,2 \mathrm{~m}$ e $40,4 \mathrm{~cm}$, respectivamente.

De cada árvore foi coletado um disco, retirado à altura do peito, para obtenção de material macerado utilizado na determinação do comprimento dos traqueídes axiais. A finalidade foi demarcar previamente a madeira juvenil e adulta. Para o estudo anatômico foram seguidas as recomendações do método modificado de Franklin (citado por Taylor, 1975) e da norma ABNT-IAWA (1994).

As observações e medições dos traqueídes foram realizadas através do analisador de imagens e microcomputador Videoplan, no Laboratório de Anatomia da Madeira da Universidade Federal do Paraná.

Para o estudo das propriedades mecânicas foram coletados dois toretes de cada árvore, com 2,20 m de comprimento cada, tomados dos primeiros $12 \mathrm{~m}$ do tronco. De cada torete foi retirado um pranchão central de $8,0 \mathrm{~cm}$ de espessura por 2,20 $\mathrm{m}$ de comprimento.

Com os resultados da análise anatômica do lenho, foram demarcadas e separadas três regiões de cada pranchão central e de cada posição, na direção radial da árvore (MJ - madeira juvenil, MA - madeira adulta e RT - região de transição). A região de transição (RT) e a região da medula foram eliminadas, evitando-se interferências nos resultados. Por fim, de cada pranchão foram confeccionados os corpos-de-prova, previamente classificados em dois grupos, de acordo com o tipo de madeira (juvenil ou adulta), considerando-se as duas posições (I e II) de 1,0 $\mathrm{m}$ de comprimento, conforme indicado na Figura 1.

Com isso, os corpos-de-prova para os ensaios de flexão $(2 \times 2 \times 46 \mathrm{~cm})$ foram retirados do pranchão central de forma sistemática, respeitando-se as regiões predefinidas anteriormente, totalizando 96 corpos-de-prova (quatro corpos-de-prova de madeira juvenil e quatro corpos-de-prova de madeira adulta por torete), resultando em 48 corpos-de-prova de madeira juvenil e 48 de madeira adulta. No geral, foram obedecidas as prescrições normativas da NBR 7190 (ABNT, 1997) e o preconizado por Rocco (1983), que fixa a relação vão-altura do corpode-prova igual a 21 .

Os corpos-de-prova foram climatizados em um ambiente normalizado $\left(20^{\circ} \mathrm{C} \pm 3{ }^{\circ} \mathrm{C}\right.$ de temperatura e $65 \% \pm 1 \%$ de umidade relativa), até atingirem a umidade de aproximadamente $12 \%$. Nessa situação, foram determinadas as densidades e, posteriormente, realizados os ensaios de flexão, com o uso de máquina universal de ensaios EMIC DL-10000-MF, servo-controlada, com capacidade de 10 toneladas, pertencente ao Laboratório de Ensaio de Materiais - FCA/UNESP.
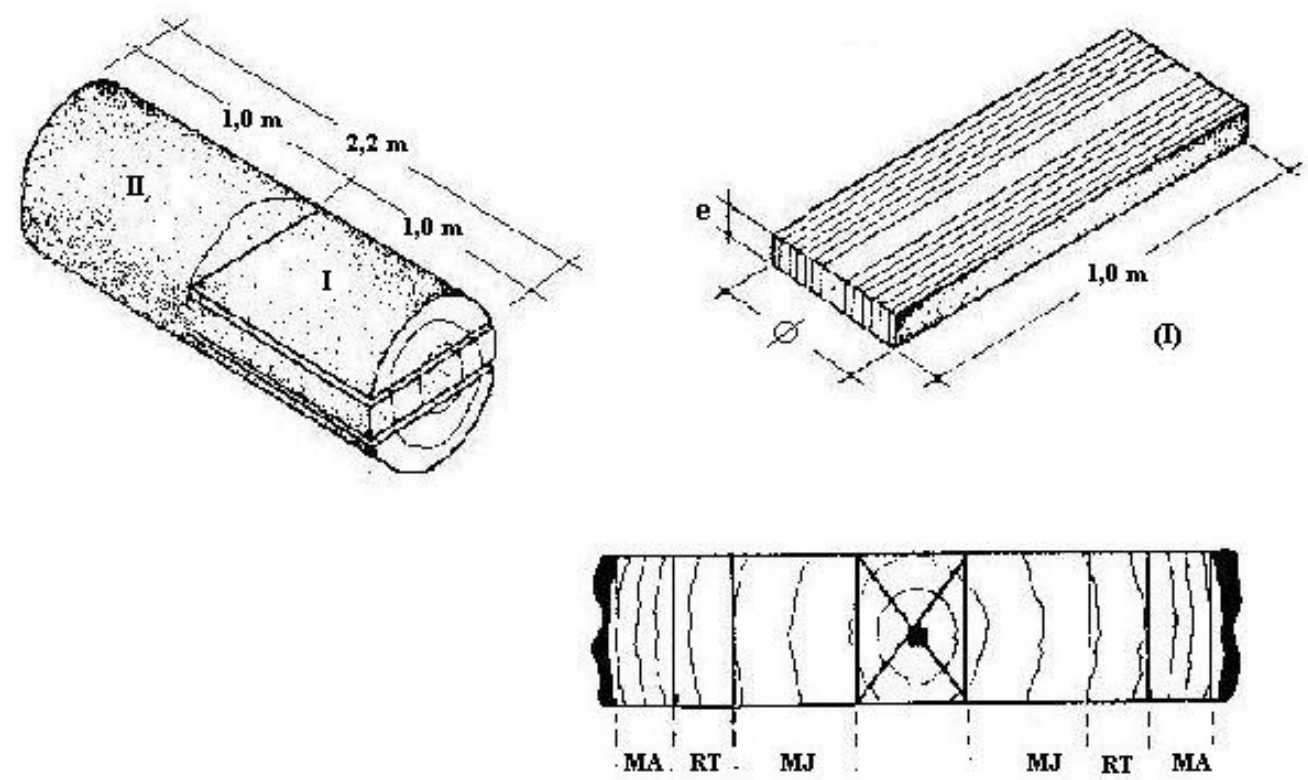

Figura 1 - Esquema da retirada do material para o estudo.

Figure 1 - Outline of the removal of the material for the study.

R. Árvore, Viçosa-MG, v.27, n.3, p.371-380, 2003 
Foram utilizados 96 corpos-de-prova para os ensaios de flexão estática (48 para madeira juvenil e 48 para madeira adulta).

Para as análises dos resultados das propriedades mecânicas, da densidade e do comprimento dos traqueídes da madeira juvenil e adulta, foram calculados os valores máximos, mínimos e médios, o desvio-padrão e o coeficiente de variação. Para avaliar a tendência do MOE e MOR na madeira, nos dois tipos de lenho, foram elaborados diagramas, cujos resultados foram plotados em ordem crescente em relação aos corpos-de-prova.

\section{RESULTADOS E DISCUSSÃO}

\subsection{Comprimento dos Traqueídes Axiais e Demarcação da Madeira Juvenil}

Na Figura 2 está apresentada a curva resultante do comprimento médio dos traqueídes axiais na direção radial, no diâmetro à altura do peito das árvores.

O comprimento dos traqueídes mostra um aumento acentuado e praticamente linear, da ordem de $66,5 \%$, até o 18 anel de crescimento. A partir do 18 o anel até o último, a taxa de aumento no comprimento diminui, tendendo para um valor mais estável e quase constante. Na Figura 2 observa-se também a regressão linear resultante com maior coeficiente de determinação $\left(R^{2}=0,96\right)$, entre o 1 e 18 anel, complementando assim a análise visual da tendência dos dados como um modo de encontrar, em forma aproximada, o limite entre a madeira juvenil e a adulta.

Essa tendência é característica na formação de madeira juvenil nos primeiros anos da árvore, comportamento este semelhante ao observado por vários autores em madeira de Pinus de crescimento rápido, tais como Senft et al. (1986), Jackson \& Megraw (1986), Bendtsen $\&$ Senft (1986) e Muñiz (1993). Os autores afirmam que a taxa de incremento no comprimento dos traqueídes é bastante rápida até os primeiros 10 a 15 anéis, diminuindo gradativamente nos anéis subseqüentes.

De acordo com este comportamento e utilizando a inspeção visual da tendência do comprimento dos traqueídes, a região compreendida entre o 14 e o 18o anel de crescimento foi considerada de transição, ficando definidas para este estudo a região de madeira juvenil desde o centro da árvore até o $14^{\circ}$ anel e a região de madeira adulta, desde o anel 18 até o anel 37, como mostra a Figura 3.

Os valores médios do comprimento dos traqueídes axiais verificados neste trabalho para as madeiras juvenil e adulta da espécie encontram-se no Quadro 1. Comparados com outros estudos apresentados na literatura para a espécie do gênero Pinus, estes foram maiores, provavelmente devido a fatores genéticos, ambientais, silviculturais.

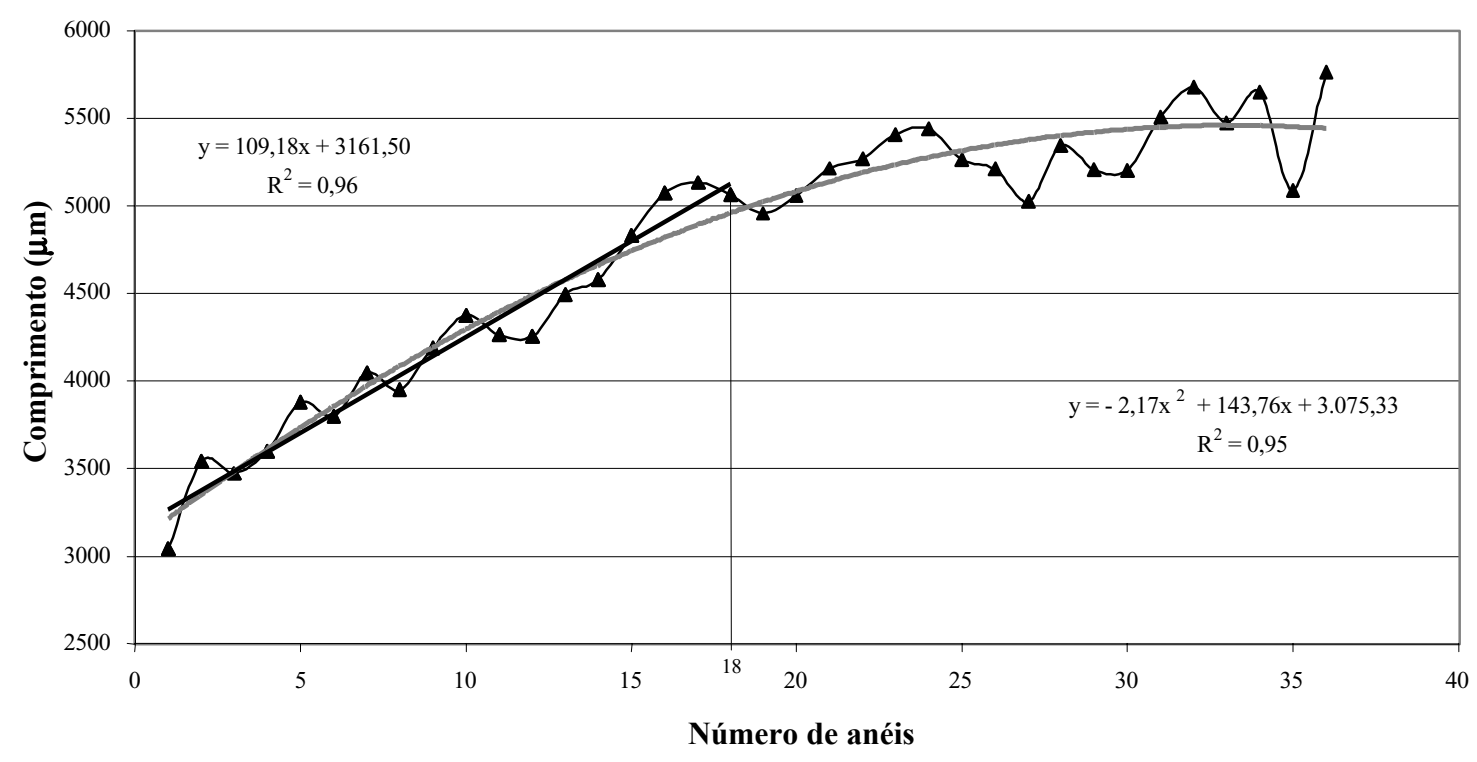

Figura 2 - Variação do comprimento dos traqueídes da madeira juvenil e adulta.

Figure 2 - Variation of tracheid length on juvenile and mature wood. 


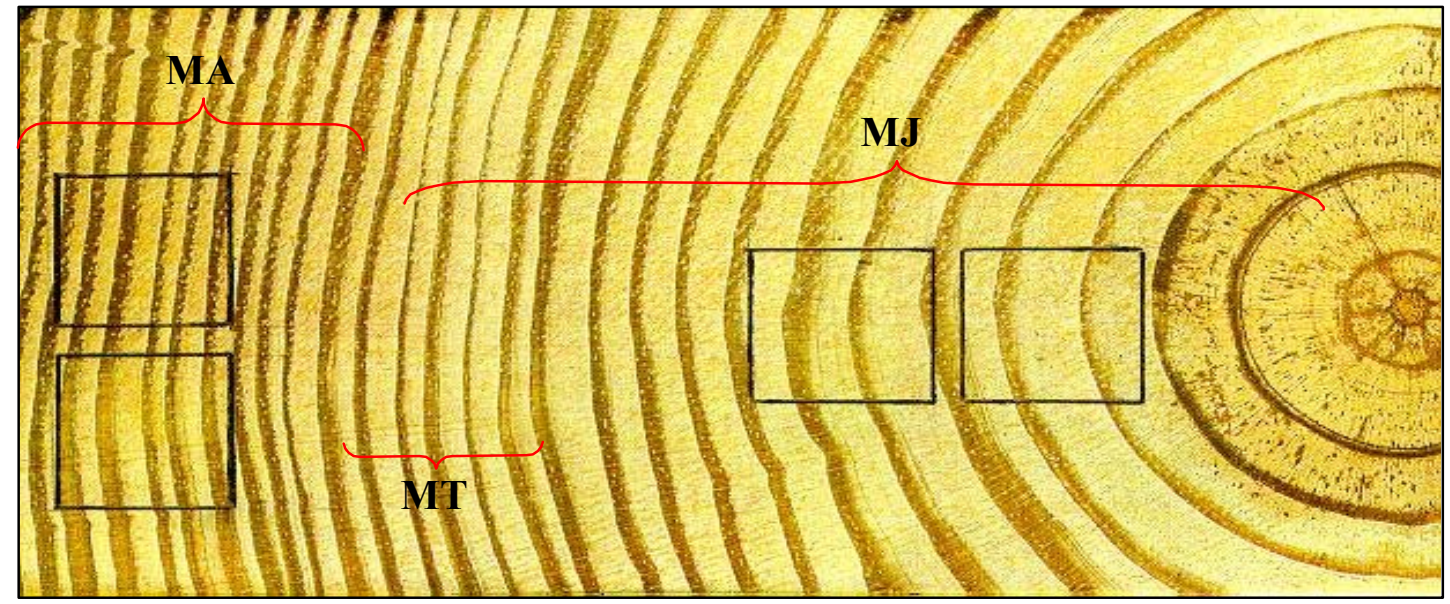

Figura 3 - Região da madeira juvenil, adulta e transição e esquema da retirada dos corpos-de-provas de flexão estática. Figure 3 - Region of juvenile, mature and transition wood and outline of the samples for static bending tests.

Quadro 1 - Comprimento dos traqueídes de Pinus taeda Table 1 - Pinus taeda tracheid length

\begin{tabular}{|l|c|c|c|}
\hline & \multicolumn{2}{|c|}{ Tipo de Madeira } & \multirow{2}{*}{ Espécie } \\
\cline { 2 - 3 } & $\begin{array}{c}\text { Madeira } \\
\text { Juvenil }\end{array}$ & $\begin{array}{c}\text { Madeira } \\
\text { Adulta }\end{array}$ & \\
\hline Comprimento máximo $(\mu \mathrm{m})$ & 7.375 & 8.325 & 8.250 \\
Comprimento mínimo $(\mu \mathrm{m})$ & 1.650 & 1.750 & 1.625 \\
Comprimento médio $(\mu \mathrm{m})$ & 4.200 & 5.322 & 4.746 \\
Desvio-padrão $(\mu \mathrm{m})$ & 1.042 & 981 & 1.158 \\
Coeficiente de variação $(\%)$ & 24,82 & 18,43 & 24,40 \\
\hline
\end{tabular}

\subsection{Propriedades Mecânicas e Densidade}

Procurando avaliar a tendência no comportamento do módulo de elasticidade e de ruptura nos dois tipos de madeira (juvenil e adulta), foram elaborados os diagramas das Figuras 4 e 5, nos quais os resultados foram plotados em relação aos corpos-de-prova. No Quadro 2 estão apresentados os resultados médios dos ensaios de flexão estática, na forma de módulo de elasticidade (MOE), módulo de ruptura (MOR) e densidade aparente a $12 \%$, obtidos dos valores individuais dos corpos-de-prova de madeira juvenil, adulta e os valores médios da espécie.

Os resultados mostram que os valores médios do MOE e MOR da madeira mais externa (adulta) foram sensivelmente maiores que os da madeira interna (juvenil). As diferenças observadas nos valores médios do MOE e do MOR na madeira adulta, em relação à juvenil, foram maiores em aproximadamente 54 e $47 \%$, respectivamente.

Segundo Cave \& Wakker (1994), a grande diferença de resistência e rigidez entre a madeira adulta e juvenil não ocorre exclusivamente pelas diferenças de densidade, sendo o maior ângulo fibrilar dos traqueídes da madeira juvenil o maior responsável por estas propriedades.

Nas Figuras 6 e 7 estão as equações de regressões, ajustadas para estimar o MOE e o MOR, em função da densidade nos dois tipos de madeira. Os modelos representam valores significativos a $5 \%$ de probabilidade. Observa-se que as regressões foram significativas, com coeficiente de correlação de 0,75 para o MOE e de 0,66 para o MOR na madeira adulta, valores estes superiores aos encontrados na madeira juvenil, que foram de $0,52 \mathrm{e}$ 0,53 , respectivamente. A diferença nas regressões obtidas para as duas madeiras, associada aos coeficientes de variação (Quadro 2), evidencia a grande variabilidade que as propriedades analisadas têm na região da madeira juvenil.

No Quadro 2 constata-se, ainda, que os valores médios de densidade obtidos para a madeira mais externa (adulta) foram sensivelmente maiores que os obtidos para a madeira interna (juvenil). As diferenças observadas nos valores médios da densidade aparente da madeira adulta em relação à juvenil foram da ordem de $26 \%$. 


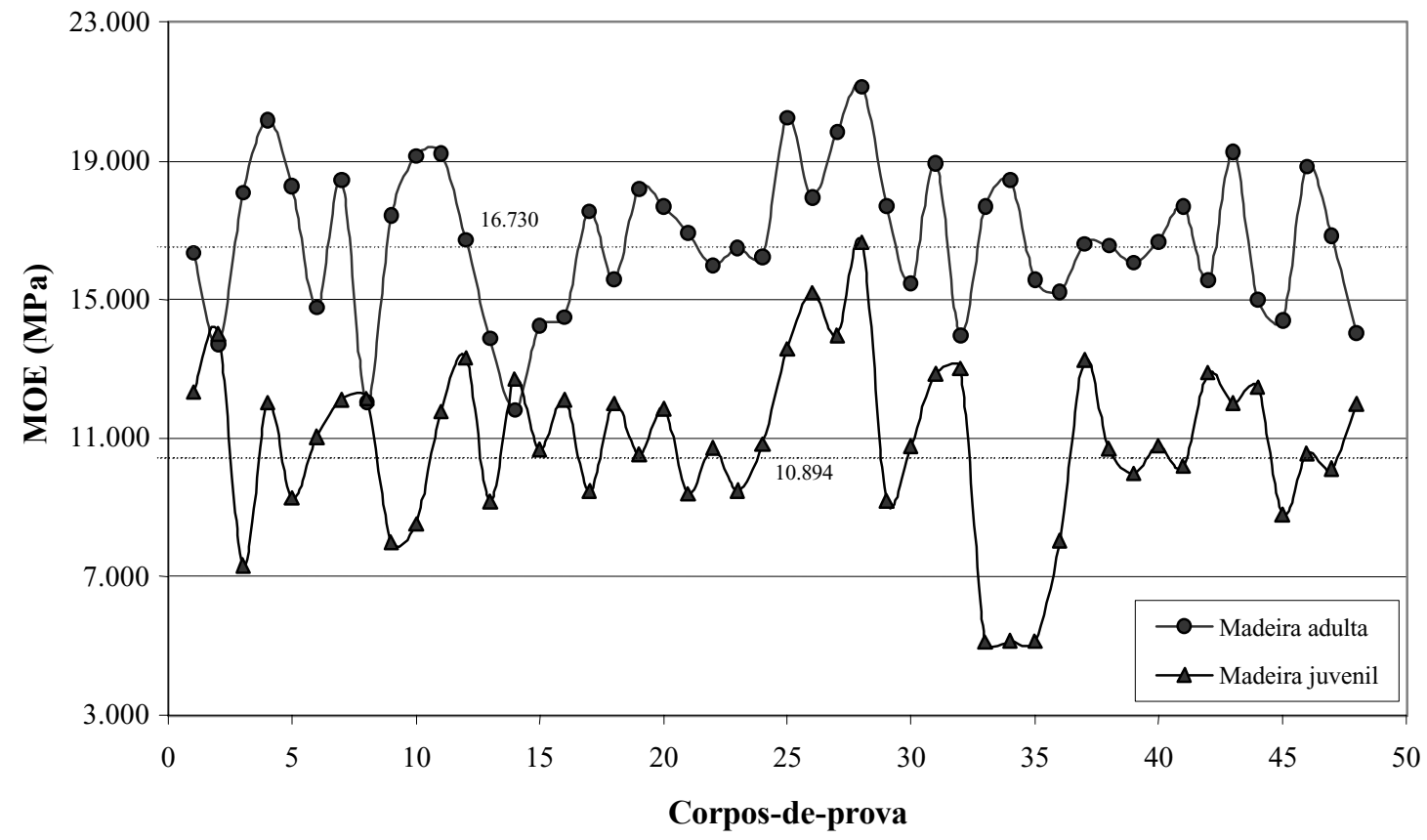

Figura 4 - Módulo de elasticidade da madeira adulta e juvenil.

Figure 4-Modulus of elasticity of mature and juvenile wood.

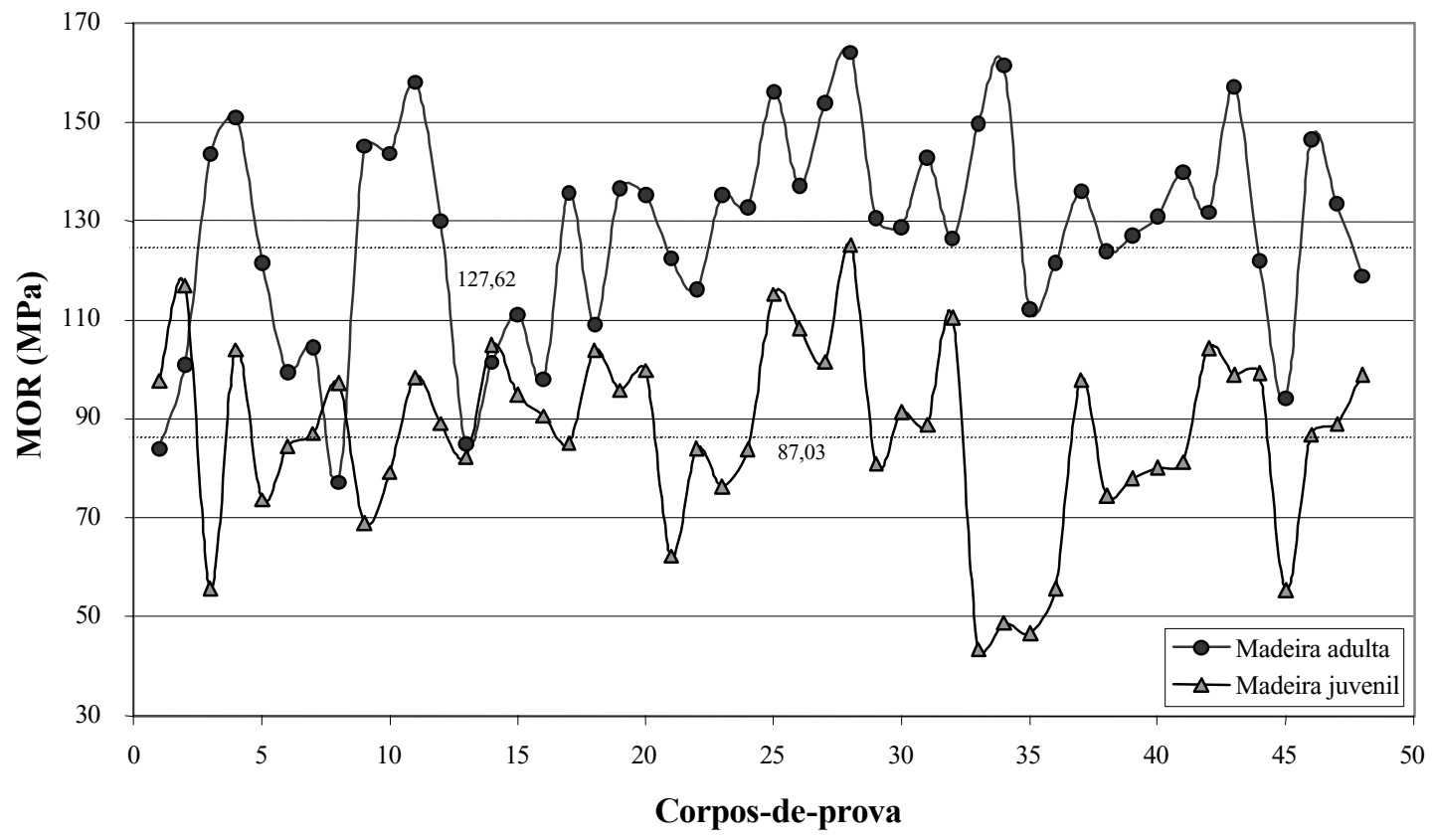

Figura 5 - Módulo de ruptura da madeira adulta e juvenil.

Figure 5 -Modulus of rupture of mature and juvenile wood. 
Quadro 2 - Valores médios do MOE, MOR e densidade da madeira

Table 2 - Average values of MOE, MOR and density of wood

\begin{tabular}{|l|c|c|c|c|c|c|c|c|c|}
\hline & \multicolumn{3}{|c|}{ Madeira Adulta } & \multicolumn{3}{c|}{ Madeira Juvenil } & \multicolumn{3}{c|}{ Espécie } \\
\cline { 2 - 10 } & $\begin{array}{c}\text { MOE } \\
(\mathrm{MPa})\end{array}$ & $\begin{array}{c}\text { MOR } \\
(\mathrm{MPa})\end{array}$ & $\begin{array}{c}\text { Dens. 12\% } \\
\left(\mathrm{g} / \mathrm{cm}^{3}\right)\end{array}$ & $\begin{array}{c}\text { MOE } \\
(\mathrm{MPa})\end{array}$ & $\begin{array}{c}\text { MOR } \\
(\mathrm{MPa})\end{array}$ & $\begin{array}{c}\text { Dens. 12\% } \\
\left(\mathrm{g} / \mathrm{cm}^{3}\right)\end{array}$ & $\begin{array}{c}\text { MOE } \\
(\mathrm{MPa})\end{array}$ & $\begin{array}{c}\text { MOR } \\
(\mathrm{MPa})\end{array}$ & $\begin{array}{c}\text { Dens. 12\% } \\
\left(\mathrm{g} / \mathrm{cm}^{3}\right)\end{array}$ \\
\hline Mínimo & 11.820 & 77,12 & 0,586 & 5.112 & 43,45 & 0,439 & 5.112 & 43,45 & 0,420 \\
Máximo & 21.139 & 164,22 & 0,776 & 16.641 & 125,08 & 0,699 & 21.139 & 43,45 & 0,787 \\
Média & 16.730 & 127,62 & 0,674 & 10.894 & 87,03 & 0,536 & 13.812 & 107,02 & 0,605 \\
CV (\%) & 12,7 & 16,6 & 7,99 & 22,3 & 21,5 & 10,7 & 26,8 & 26,6 & 14,63 \\
$\mathrm{~s}$ & 2.117 & 21,2 & 0,054 & 2.426 & 18.71 & 0,058 & 3706 & 28,49 & 0,089 \\
\hline
\end{tabular}

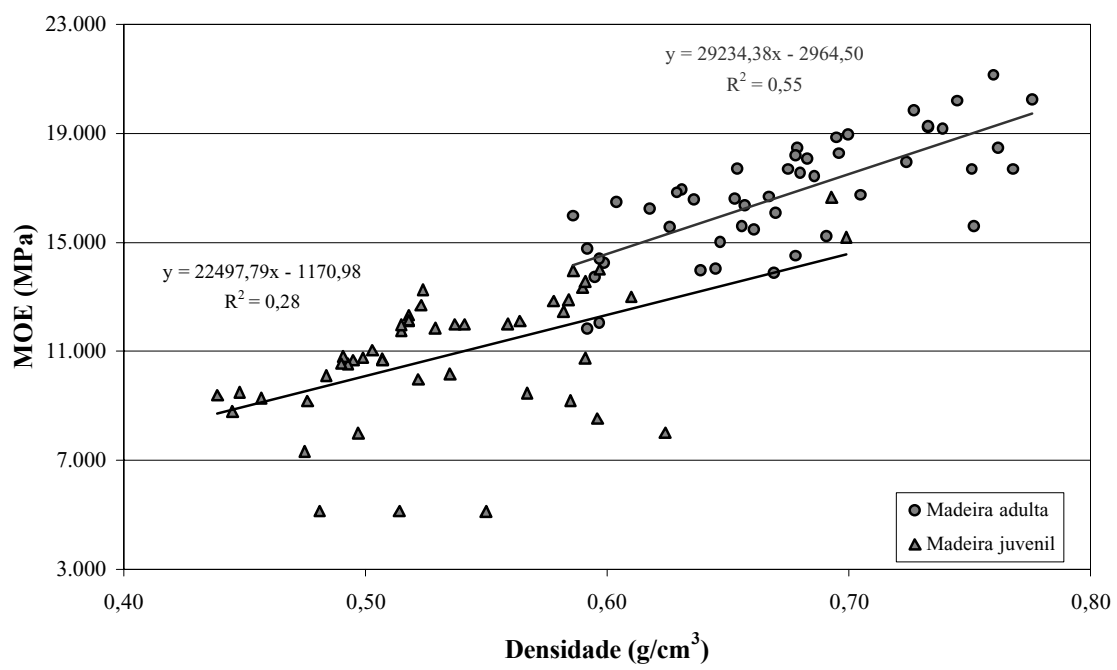

Figura 6 - Relação entre o módulo de elasticidade e a densidade.

Figure 6 -Relationship between modulus of elasticity and density.

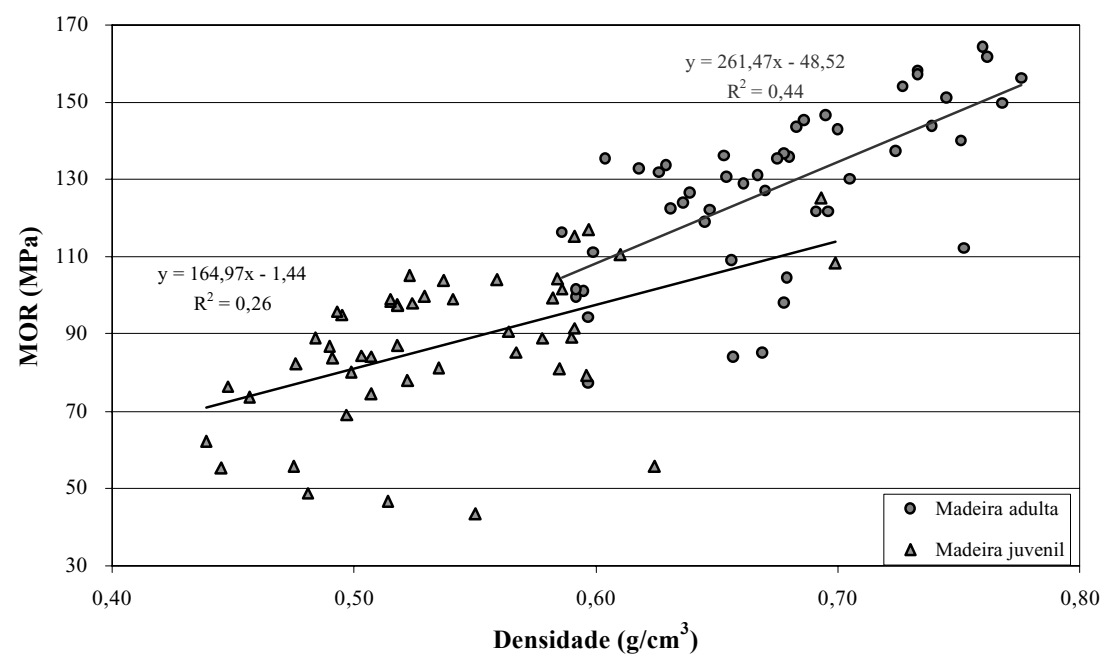

Figura 7 - Relação entre o módulo de ruptura e a densidade.

Figure 7 - Relationship between modulus of rupture and density.

R. Árvore, Viçosa-MG, v.27, n.3, p.371-380, 2003 
A variação da densidade apresentada para a espécie $(\mathrm{CV}=14,63 \%)$ foi superior à apresentada pelas madeiras juvenil e adulta. Em geral, a alta variação da densidade na árvore e entre árvores é característica do gênero Pinus, e se deve a vários fatores (ambientais, genéticos, silviculturais, madeira juvenil etc.), podendo chegar a aproximadamente $30 \%$ da média da espécie, segundo Trendelenburg, citado por Muñiz(1993).

\section{CONCLUSÕES}

Com base nos resultados apresentados para as propriedades da madeira de Pinus taeda L. estudadas, pode-se concluir:

- O comprimento dos traqueídes aumentou na direção radial da árvore, no sentido medulacasca.

- Os traqueídes tiveram maior aumento no comprimento, nos primeiros 18 anéis.

- Do $20^{\circ}$ anel em diante, os comprimentos dos traqueídes apresentaram pouca variação.

- A tendência apresentada no comprimento dos traqueídes axiais confirmou o padrão de variação no sentido transversal ao tronco, em espécies de rápido crescimento do gênero Pinus.

- As propriedades de resistência e rigidez à flexão estática foram superiores e menos variáveis na madeira adulta do que na madeira juvenil.

- Os valores dos módulos de elasticidade e de ruptura da flexão estática mostraram correlações mais significativas na madeira adulta do que na madeira juvenil.

- Os módulos de elasticidade e de ruptura sofreram maior influência da densidade na madeira adulta.

- A densidade aparente da madeira adulta foi maior do que na madeira juvenil.

\section{REFERÊNCIAS BIBLIOGRÁFICAS}

ABDEL-GADIR, A.Y.; KRAHMER, R. L. Estimating the age of demarcation of juvenile and mature wood in Douglasfir. Wood and Fiber Science, v. 25, n. 3, p. 242-249, 1993.

ASSOCIAÇÃO BRASILEIRA DE NORMAS TÉCNICAS ABNT. Projeto de estruturas de madeira ABNT (NBR-

7190). Rio de Janeiro: 1997. 107 p.
ASSOCIAÇÃO BRASILEIRA DE NORMAS TÉCNICAS. INTERNATIONAL ASSOCIATION OF WOOD

ANATOMISTS. ABNT - IAWA. Leiden: 1994.

BENDTSEN, B. Properties of wood from improved and intensively managed trees. Forest Production Journal, v. 28, n. 10 , p. $61-72,1978$.

BENDTSEN, B.; SENFT, J. Mechanical and anatomical properties in individual growth rings of plantation-grown eastern Cottonwood and Loblolly Pine. Wood and Fiber Science, v. 18, n. 1, p. 21-38, 1986.

BROWN, M. J.; McWILLIAMS, W. H. Pine stands across the South - trends and projections. In: SOUTHERN PLANTATION WOOD QUALITY WORKSHOP, 1989, Athens. Proceedings... Asheville: Southeastern Forest Experiment Station, United States Department of Agriculture, 1990. p. 1-15.

CAVE, I. D.; WALKER, J. C. F. Stiffness of wood in fastgrown plantation softwoods: the influence of microfibril angle. Forest Productions Journal, v. 44, n. 5, p. 43-48, 1994.

CLARK, A. III.; SAUCIER, J. R. Influence of initial planting density, geographic location, and species on juvenile wood formation in southern pine. Forest Productions Journal, v. 39, n. 7/8, p. 42-48, 1989.

COWN, D. J. Corewood (Juvenile wood) in Pinus radiata should we be concerned?. New Zealand Journal of Forestry Science, v. 22, n. 1, p. 87-95, 1992.

EVANS II, J. W.; SENFT, J. F.; GREEN, D. W. Juvenile wood effect in red alder: analysis of physical and mechanical data to delineate juvenile and mature wood zones. Forest Productions Journal, v. 50, n. 7/8, p. 75-87, 2000.

GEIMER, R. L.; HERIAN, V. L.; XU, D. Influence of juvenile wood on dimensional stability and tensile properties of flakeboard. Wood and Fiber Science, v. 29, n. 2, p. 103$120,1997$.

JACKSON, M.; MEGRAW, R. A. Impact of juvenile wood on pulp and paper products. In: A TECHNICAL WORKSHOP: JUVENILE WOOD-WHAT DOES IT MEAN TO FOREST MANAGEMENT AND FOREST PRODUCTS, 1985, Washington. Proceedings... Madison: Forest Products Research Society, 1986. p. 75-81.

KRAHMER, R. Fundamental anatomy of juvenile and mature wood. In: A TECHNICAL WORKSHOP: JUVENILE WOOD-WHAT DOES IT MEAN TO FOREST MANAGEMENT AND FOREST PRODUCTS, 1985, Washington. Proceedings... Madison: Forest Products Research Society, 1986. p. 12-16.

R. Árvore, Viçosa-MG, v.27, n.3, p.371-380, 2003 
KRETSCHMANN, D. E. Effect of juvenile wood on shear parallel and compression perpendicular-to-grain strength for loblolly pine. In: CTIA/IUFRO INTERNATIONAL WOOD QUALITY WORKSHOP, 1997, Quebec. Proceedings... Quebec: Forintek Canada, 1997. p. 23-30.

KRETSCHMANN, D. E.; BENDTSEN, B. A. Ultimate tensile stress and modulus of elasticity of fast-grown plantation Loblolly Pine lumber. Wood and Fiber Science, v. 24, n. 2, p. 189-203, 1992.

LARSON, P. R. et al. Formation and properties of juvenile wood in southern pines - a synopsis. Madison: U.S. Department of Agriculture, Forest Service, Forest Products Laboratory, 2001. FPL Reporter

LOO, J. A.; TAUER, C. C.; McNEW, R. N. Genetic variation in the time of transition from juvenile to mature wood in Loblolly Pine (Pinus taeda). Silvae Genet, n. 34, p. 14-19, 1985.

McALISTER, R.; CLARK, A. Effect of geographic location and seed source on the bending properties of juvenile and mature Loblolly Pine. Forest Productions Journal, v. 41, n. 9, p. 39-41, 1991.

McALISTER, R.; CLARK, A.; SAUCIER, J. Effect of initial spacing on mechanical properties of lumber sawn from unthinned Slash Pine at age 40. Forest Productions Journal, v. 47, n. 7/8, p. 107-109, 1997.

MUÑIZ, G. I. Caracterização e desenvolvimento de modelos para estimar as propriedades e comportamento na secagem da madeira de Pinus elliottii Engelm. e Pinus taeda L. 1993. 236 f. Tese (Doutorado em Ciências Florestais) - Universidade Federal do Paraná, Curitiba, 1993.

PEARSON, R. G. Compressive properties of clear and knotty Loblolly Pine juvenile wood. Forest Productions Journal, v. 38, n. 7/8, p. 15-22, 1988.

PEARSON, R. G.; ROSS, B. E. Growth rate and bending properties of selected Loblolly Pines. Wood and Fiber Science, n. 16, p. 37-47, 1984.

RAMSAY, W.; BRIGGS, D. Juvenile wood: has it come of age?. In: A TECHNICAL WORKSHOP: JUVENILE WOOD-WHAT DOES IT MEAN TO FOREST MANAGEMENT AND FOREST PRODUCTS, 1985, Washington. Proceedings... Madison: Forest Products Research Society, 1986. p. 5-11.

ROCCO, F. A. Sobre a determinação de propriedades de elasticidade da madeira. 1983. $221 \mathrm{f}$. Tese (Doutorado em Engenharia de Estruturas) - Universidade de São Paulo, São Carlos, 1983.

R. Árvore, Viçosa-MG, v.27, n.3, p.371-380, 2003
ROSS, K. D.; SHOTTAFER, J. E.; SHEPARD, R. K. The relationship between selected mechanical properties and age in Quaking aspen. Forest Productions Journal, v. 40, n. $7 /$ 8, p. 54-56, 1990.

ROWELL, R. M.; HAN, J. S.; ROWELL, J. S. Characterization and factors affecting fiber properties. In: FROLLINI, E.; LEÃO, A. L.; MATTOSO, L. H. C. (Eds). Natural polymers and agrofibers composites: São Carlos: IQSC, USP, 2000. p. 115-133.

SENFT, J. F.; QUANCI, M. J.; BENDTSEN, B. A. Property profile of 60-year-old Douglas - Fir. In: A TECHNICAL WORKSHOP: JUVENILE WOOD-WHAT DOES IT MEAN TO FOREST MANAGEMENT AND FOREST PRODUCTS, 1985, Washington. Proceedings... Madison: Forest Products Research Society, 1986. p. 17-28.

SENFT, J. F.; BENDTSEN, B. A.; GALLIGAN, W. L. Weak wood. Journal of Forestry, n. 83, p. 476-485, 1985.

TASISSA, G.; BURKHART, H. E. Juvenile-mature wood demarcations in Loblolly Pine trees. Wood and Fiber Science, v. 30, n. 2, p. 119-127, 1998.

TAYLOR, F. W. Fiber length mensurement - an accurate inexpensive technique. Tappi, v. 58, n. 12, p. 126-127, 1975.

TOMASELLI, I. Planted forests in Brazil. In: PLANTED FORESTS IN SARAWAK, AN INTERNATIONAL CONFERENCE, 1998, Sarawak. Proceedings... Sarawak: Kuching Forest Department, 1998. p. 2-35.

YANG, K. C.; BENSON, C. A.; WONG, J. K. Distribution of juvenile wood in two stems of Larix laricina. Canadian Journal Forest Research, v. 16, p. 1041-1049, 1986.

YANG, K. C.; HAZENBERG, G. Impact of spacing on tracheid length, relative density, and growth rate of juvenile wood and mature wood in Picea mariana. Canadian Journal Research, v. 24, p. 996-1007, 1994.

ZOBEL, B. J.; BUIJTENEN, J. P. Wood variation: its causes and control. Berlin: Springer-Verlag, 1989. 361 p.

ZOBEL, B. J. Genetic manipulation of wood of the southern pines including chemical characteristics. Wood Science Technology, n. 54, p. 255-271, 1971.

ZOBEL, B. J. Inherent differences affecting wood quality in fast-grown plantations. In: IUFRO CONFERENCE, Oxford. Proceedings... Oxford: IUFRO 1980. p. 169-188.

ZOBEL, B. Wood quality from fast-grown plantations. Tappi, v. 64, n. 1, p.71-74, 1981.

ZOBEL, B. J. The changing quality of the world wood supply. Wood Science Technology, n. 18, p. 1-17, 1984. 